\title{
CAPACIDADE DE ABSORÇÃO DE EMPRESAS QUE INTERAGEM COM UNIVERSIDADES: UMA ANÁLISE QUALITATIVA APLICANDO CONJUNTOS FUZZY.
}

\author{
Júlio Eduardo Rohenkohl ${ }^{1}$ \\ Andreia Cunha da Rosa ${ }^{2}$ \\ Orlando Martinelli ${ }^{3}$ \\ Janaína Ruffoni ${ }^{4}$
}

\begin{abstract}
Resumo
$\mathrm{O}$ artigo analisa quais os atributos da capacidade de absorção elevada de empresas que interagem com universidades. Os dados de um survey com empresas que possuem interação com universidade são analisados com a teoria dos conjuntos fuzzy. O modelo analítico de capacidade de absorção da firma incorpora contribuições teóricas importantes ao explorar as noções de capacidade de aquisição, assimilação, transformação e exploração como condições antecedentes para o estabelecimento da capacidade de absorção, permitindo a análise comparativa dos dados através de pertencimento a conjuntos qualitativos. O uso do software de processamento de dados fsQCA permitiu testar condições necessárias e suficientes qualitativamente distintas para as empresas da amostra alcançarem uma elevada capacidade de absorção. Encontraram-se duas combinações causais consistentes e abrangentes. O conjunto capacidade de "assimilação alta" destaca-se como requisito muito relevante para as empresas obterem alta capacidade de absorção.
\end{abstract}

Palavras-chave: Interação Universidade-Empresa, Capacidade de absorção da firma, Conjuntos fuzzy

JEL: O30, L60

Área temática: 5.7 Competências e capacitações das empresas

\footnotetext{
${ }^{1}$ Departamento de Economia e Relações Internacionais da Universidade Federal de Santa Maria

${ }^{2}$ Programa de Pós-Graduação em Economia da UNISINOS

${ }^{3}$ Programa de Pós-Graduação em Economia e Desenvolvimento da Universidade Federal de Santa Maria

${ }^{4}$ Programa de Pós-Graduação em Economia da UNISINOS
} 


\title{
ABSORPTION CAPACITY OF COMPANIES THAT INTERACT WITH UNIVERSITIES: A QUALITATIVE ANALYSIS APPLYING FUZZY SETS.
}

\begin{abstract}
The article analyzes the attributes of the high absorption capacity of firms that interact with universities. The data of a survey with companies that have interaction with university are analyzed with the theory of fuzzy sets. The firm's analytical capacity absorption model incorporates important theoretical contributions by exploring the notions of acquisition capacity, assimilation, transformation and exploitation as antecedent conditions for the establishment of absorption capacity, allowing the comparative analysis of the data through membership of qualitative sets. The use of the fsQCA data processing software allowed us to test necessary and qualitatively different conditions for the firms of the sample to reach a high absorption capacity. Two consistent and comprehensive causal combinations were found. The set of "high assimilation" capacity stands out as a very relevant requirement for firms to obtain high absorption capacity
\end{abstract}

Key-words: University-firms interactions, firms' absorptive capacity, fuzzy set.

JEL: O30, L60 


\section{Introdução}

Alguns estudos têm reconhecido que uma mesma inovação, quando introduzida em diferentes organizações, gera resultados diferentes dependendo do contexto no qual é inserida e das ações das principais partes interessadas durante e depois da implementação (Barley, 1986; Orlikowski, 1993; Robey e Sahay, 1996; Markus e Robey, 1988; Ciborra, 2000 apud Cullar e Gallivan, 2006). Conforme Spender (1989), diferenças na trajetória das empresas e seus recursos, particularmente o conhecimento, leva cada uma delas a desenvolver um conjunto exclusivo de capacidades para realizar suas atividades.

Quando uma empresa deseja adquirir e usar conhecimentos externos a ela e que não estão relacionados com sua base de conhecimento atual, esforços deliberados são necessários para esse propósito. Nesse sentido, a literatura acadêmica destaca importantes contribuições da interação entre universidade e empresa (UE), ao permitir que as firmas consigam, por meio da troca de conhecimentos (lato sensu) com o meio acadêmico, incrementar seus desempenhos inovativos (Link e Rees, 1990; Mansfield, 1991; George e Zahra et al., 2002; Laursen e Salter, 2004; Póvoa, 2008).

O conceito de Capacidade de Absorção (CA) é definido por Cohen e Levinthal $(1989 ; 1990)$ como a capacidade de uma organização de reconhecer o valor do novo conhecimento externo, assimilá-lo e aplicá-lo para fins comerciais. Para esses autores, empresas com níveis mais elevados de capacidade de absorção tendem a ser mais proativas e capazes de explorar as oportunidades presentes no ambiente, componente fundamental da capacidade inovadora de uma organização. Para compreender além do nível geral de CA da firma, é necessário considerar as diferentes dimensões que a compõem.

$\mathrm{O}$ artigo analisa quais os atributos da capacidade de absorção de empresas que interagem com universidades. A partir da teoria dos conjuntos fuzzy é construído um modelo analítico que incorpora e hierarquiza os graus da capacidade de absorção, permitindo a análise comparativa dos dados através de pertencimento a conjuntos qualitativos, utilizando-se a teoria dos conjuntos fuzzy ${ }^{5}$. As variáveis linguísticas (palavras ou sentenças) podem assumir valores, cujos graus de precisão não podem ser mensurados com certeza. A CA é definida por um conjunto de outras variáveis linguísticas. Neste trabalho, essas variáveis são: a capacidade de aquisição, a capacidade de transformação, e a capacidade de exploração do conhecimento pela empresa. É difícil definir com precisão qual o nível de CA que a empresa detém, assim pode ser mais relevante e analiticamente mais robusto construir relações condicionais $f u z z y$, que possibilitem a identificação de condições - e de graus de pertencimento - em que as empresas possam estar associadas a diferentes níveis de CA.

Assim, diferentemente de trabalhar com conjuntos a partir de variáveis binárias (característico de crisp set - Booleano), o uso de conjuntos fuzzy permite inserir valores de associação entre ' 0 ' (nenhuma associação) e ' 1 ' (total associação) a um conjunto - e não somente ' 0 ' ou' 1 ' - ampliando as situações que se pretende analisar. Por exemplo: uma situação X (e.g. a determinação do grau de capacidade de absorção da empresa) pode ter diferentes graus de pertencimento a uma condição A (e.g. os diferentes tipos relacionamentos com grupos de pesquisa de universidades).

O trabalho está organizado em cinco partes, incluindo essa introdução. Na segunda apresenta-se a base conceitual sobre capacidade absortiva. Na terceira parte aprofunda-se a parte metodológica, tanto da forma de coleta dos dados, mas também do método de análise. Na quarta é feita a descrição e análise dos dados. Por fim, são feitas algumas considerações.

\footnotetext{
${ }^{5}$ Diferentemente de modelos econométricos baseados em critérios binários (0 ou 1$)$ de classificação das variáveis, a teoria dos conjuntos fuzzy permite lidar com problemas em que a imprecisão não resulta do comportamento aleatório das variáveis, mas da ausência de critérios claramente definidos de pertencimento (de uma observação ou indivíduo) a um determinado conjunto.
} 


\section{Revisão Teórica: interação universidade-empresa e capacidade de absorção de empresas}

Entre as diversas iniciativas empresariais para dar conta do dinamismo e da concorrência em mercado globalizado e mutante, destaca-se a aproximação entre empresas e universidades. Essa aproximação se refere à relação baseada na transferência de conhecimento, no qual atores públicos e privados cooperam conjuntamente com recursos financeiros, humanos e de infraestrutura envolvidos no empreendimento. (GUSMÃO, 2002).

Nelson (1986) apresenta a importância das universidades e institutos de pesquisa para o processo de inovação industrial e concluiu que muitas áreas da ciência são importantes para determinados setores industriais. $\mathrm{O}$ autor destaca que a pesquisa oriunda das universidades, raramente gera nova tecnologia, mas antes disso, ela age de forma indireta, potencializando as oportunidades tecnológicas das firmas. Desta forma, a interação universidade-empresa torna-se fundamental no sentido da possibilidade da aplicação das pesquisas geradas nas universidades, podendo resultar no aumento da capacidade das empresas de gerar inovações.

No Brasil, conforme estudo de Rapini e Righi (2011), a relação entre universidades e empresas caracteriza-se por 'manchas de interação', ou seja, há interações entre esses dois atores do sistema de inovação, mas essas não estão presentes de forma homogênea e generalizada. $\mathrm{O}$ estudo identificou 18 manchas de interação no território nacional, sendo 6 na área de Engenharia de Materiais e Metalúrgica e 4 na de Agronomia. O setor de Produtos Químicos apresentou o maior número de manchas, de acordo com a Classificação Nacional de Atividade Empresarial (CNAE). Conforme as autoras, a interação universidades-empresa está concentrada nos estados das regiões Sul e Sudeste, sendo São Paulo considerado em primeiro lugar, com 465 grupos de pesquisa com interação e o Rio Grande do Sul considerado o segundo estado com maior número de grupos de pesquisa com interação (265).

No caso do Rio Grande do Sul, Costa et al. (2011) mencionam que, dos 2.072 grupos de pesquisa do RS cadastrados no Diretório de Grupos de Pesquisa (DGP) do CNPq em 2004, 265 (12\%) afirmaram ter algum tipo de relacionamento com um total de 430 unidades do setor produtivo, gerando uma densidade média de interação de 1,6. Com isso o RS foi identificado como o segundo estado do país com maior número de grupos de pesquisa interagindo com o setor produtivo.

De acordo com Abramovsky et al. (2009), a capacidade das empresas para aproveitarem os conhecimentos gerados externamente tem um efeito positivo sobre o sucesso das inovações e está associada com a decisão em realizar a pesquisa em colaboração formal com outras instituições. Com isso, a capacidade de uma firma de absorver conhecimentos do ambiente externo (como os gerados em Universidades) e usá-los no desenvolvimento de inovações, referida como a capacidade de absorção, tornou-se uma importante questão nos estudos da área de economia industrial. (WAALKENS, 2006).

O conceito de "capacidade de absorção" da empresa foi inicialmente desenvolvimento por Cohen e Levinthal $(1989,1990,1994)$. Entretanto, sua origem está enraizada em publicações anteriores na área de economia do desenvolvimento. A capacidade de absorção tem muito em comum com a noção de capacidade social utilizada no contexto dos países em desenvolvimento, para melhorar as suas capacidades (tais como: competências técnicas, instituições e mercados financeiros, a estabilidade do governo, honestidade e confiança) que lhes permitissem um processo de catching-up com economias mais desenvolvidas (ABRAMOVITZ, 1986).

Conforme Lewandowska (2015), a capacidade de absorção foi amplamente entendida como a capacidade de países em desenvolvimento absorverem novos investimentos. Mais tarde, como o papel do conhecimento tornou-se mais importante para o crescimento e desenvolvimento econômico dos países, a capacidade de absorção também foi compreendida como a "capacidade de 
absorver conhecimentos". Assim, o conceito de capacidade social e capacidade de absorção foram primeiramente associados com o nível de economia nacional. Posteriormente, muitos pesquisadores buscaram compreender qual a capacidade da empresa para adquirir conhecimento a partir de seu ambiente externo (TILTON, 1971; ALLEN, 1984 apud LEWANDOWSKA, 2015).

Foi a partir do trabalho seminal de Cohen e Levinthal (1989) que se disseminaram diversos estudos sobre a capacidade de absorção das empresas. Cohen e Levinthal (1989), argumentaram que empresas com níveis mais elevados de capacidade de absorção tendem a ser mais proativas e capazes de explorar as oportunidades presentes no ambiente, componente fundamental da capacidade inovadora de uma organização. Conforme eles, a capacidade de absorção leva a um maior aprendizado, aumentando a base de conhecimento externo da empresa, que por sua vez, aumenta a capacidade de absorção. Por conta disso, o trabalho aqui desenvolvido objetiva identificar atributos que as empresas que interagem com universidades e desenvolvem capacidade absortiva alta consideram importantes e descrever como combinam tais componentes.

O conceito de Capacidade de Absorção (CA) é definido por Cohen e Levinthal $(1989,1990)$ como a capacidade de uma organização de reconhecer o valor do novo conhecimento externo, assimilá-lo e aplicá-lo para fins comerciais. Esses mesmos autores, consideram que a inovação é gerada por meio do processo de aprendizado da firma (dependente de trajetória) e que a capacidade de absorção é constituída a partir de um conjunto de conhecimentos prévios. Os autores argumentam que a capacidade de avaliar e utilizar o conhecimento externo é em grande parte função do nível de conhecimento prévio. No nível mais elementar, este conhecimento prévio inclui habilidades básicas ou até mesmo uma linguagem comum, mas pode também incluir o conhecimento dos mais recentes desenvolvimentos científicos ou tecnológicos de uma determinada área. Essas habilidades coletivas constituem o que os autores denominam de 'Capacidade de Absorção'.

Cohen e Levinthal (1990) sugerem que empresas com níveis mais elevados de capacidade de de absorção tendem a ser mais proativas e capazes de explorar as oportunidades presentes no ambiente. Desta forma, a relação entre o aprendizado organizacional e a capacidade absortiva é o ponto central dos estudos elaborados por Cohen e Levinthal $(1989,1990)$, no qual a CA leva a um maior aprendizado, aumentando a base de conhecimento externo da empresa, que por sua vez, aumenta a capacidade absortiva e, com isso, leva a um maior potencial de aprendizado.

A definição original de capacidade absortiva elaborada por Cohen e Levinthal $(1989,1990)$ destaca três dimensões - identificação do conhecimento, assimilação, e exploração para fins comerciais - mas várias re-conceituações têm surgido na literatura (por exemplo, Jansen et al, 2005; Torodova \& Durisin, 2007; Van Den Bosch et al, 2003; Zahra \& George, 2002), pois se entendia que o conceito estava sendo utilizado mais como uma retórica do que como uma dimensão investigativa (Versiani et all, 2010). Camisón \& Forés (2009) destacam que as contribuições mais significativas na discussão da aplicação do conceito são as de Mowery e Oxley (1995), Kim (1998), Lane e Lubatkin (1998), Dyer e Singh (1998), Van den Bosch et al. (1999), Zahra e George (2002) e Lane et al. (2006).

$\mathrm{O}$ aprofundamento do conceito de capacidade de absorção ocorreu aproximadamente uma década após o seu surgimento, a partir da re-conceituação oferecida por Zahra e George (2002), ao definir a CA como um conjunto de rotinas organizacionais e de processos estratégicos pelos quais a empresa adquire, assimila, transforma e explora o conhecimento visando a criação de valor. Esses autores sugerem que a CA pode ser dividida em dois conjuntos: CA potencial e CA realizada. A capacidade absortiva potencial permite a organização ser receptiva ao conhecimento externo, isto é, adquirir, analisar, interpretar e compreender este conhecimento. Envolve as dimensões de aquisição e assimilação de conhecimento. Os autores mencionam que o simples fato de uma organização adquirir e avaliar o conhecimento externo não garante que ela consiga explorar esse conhecimento, pois isso, conforme Cohen e Levinthal (1990), depende da capacidade técnica da empresa. Mencionam que quanto maior for a capacidade técnica de uma empresa, maior 
será sua capacidade de compreender e assimilar o conhecimento externo e maiores serão os benefícios a serem obtidos a partir desse conhecimento.

Zahra e George (2002) mencionam que a capacidade absortiva realizada reflete a capacidade da empresa para transformar e explorar o novo conhecimento, incorporando-o, com o conhecimento existente, em suas operações. Esta capacidade é determinada pelas dimensões de transformação e de exploração do conhecimento. Nesta linha, dentre as contribuições mais recentes, destaca-se a de Jiménez-Barrionuevo et all (2011), no qual, a partir das definições de Zahra e George (2002) e de uma revisão bibliográfica definem as quatro dimensões da capacidade absortiva, da seguinte forma:

- $\quad$ Capacidade de aquisição: é a capacidade de uma empresa de localizar, identificar, avaliar e adquirir conhecimento externo que é importante para o desenvolvimento de suas operações (por exemplo, Lane e Lubatkin, 1998; Zahra e George, 2002).

- $\quad$ Capacidade de assimilação: é a capacidade de uma empresa de compreender o conhecimento (ou informação) trazida de fora da organização. Ou seja, é a capacidade de analisar, classificar, processar, interpretar e, finalmente, internalizar e compreender esse conhecimento (por exemplo, Cohen e Levinthal, 1990; Szulanski, 1996).

- $\quad$ Capacidade de transformação: é a capacidade de uma empresa de facilitar a transferência e combinação de conhecimento prévio com o conhecimento recém-adquirido ou assimilado. Ele consiste em adicionar ou eliminar conhecimento e de interpretar e combinar o conhecimento existente em uma maneira nova e diferente (por exemplo, Jansen et al, 2005; Todorova e Durisin, 2007).

- $\quad$ Capacidade de exploração: é a capacidade de uma empresa para incorporar o conhecimento adquirido, assimilado e transformado em suas operações e rotinas para a aplicação e uso da empresa. Esta capacidade vai dar origem à criação ou melhoria de novos produtos, sistemas, processos, formas de organização e competências (por exemplo, Lane et al, 2001; Zahra e George, 2002).

A discussão da literatura nesta área avança nas formas de mensuração da capacidade de absorção. Versiani et all (2010) e Flatten et all (2011) mencionaram que embora um número considerável de estudos empíricos tenha sido realizado sobre capacidade absortiva, uma medida válida que incorporasse suas várias dimensões ainda não tinha sido desenvolvida. A dificuldade na definição de métricas ocorre como resultado de um não consenso a respeito das dimensões que compõem o conceito. Grande parte dos pesquisadores optou em medir a CA considerando- a apenas como uma proxy de P\&D (por exemplo, Cohen e Levinthal, 1989, 1990), não incluindo suas várias dimensões e suas implicações para os diferentes resultados na firma. Desta forma, os atributos de conhecimento têm sido ignorados, apesar de sua importância ser mencionada seguidamente na literatura a respeito da transferência de tecnologia e aprendizagem da firma (VEGA-JURADO, GUTIÉRREZ-GRACIA; FERNÁNDEZ-DE- LUCIO, 2008).

Objetivando avançar nessas questões, Rosa e Ruffoni (2014) realizaram uma pesquisa bibliográfica em diferentes bases de dados, utilizando as palavras chaves "Absorptive Capacity", "Measure" e "Metrics" para o período compreendido entre 2000 e 2012. A partir desta revisão, as autoras identificaram importantes contribuições de estudos mais recentes. O levantamento resultou nos artigos elaborados por Camisón e Forés (2010), por JiménezBarrionuevo et all (2011) e por Flatten et all (2011). A partir disso, Rosa e Ruffoni (2014) desenvolveram uma proposta tendo como objetivo organizar diferentes indicadores que servem para mensurar a capacidade absortiva de empresas que interagem com universidades. A proposta das autoras procurou agrupar os itens de avaliação da CA nas dimensões de aquisição, assimilação, transformação e exploração, considerando a divisão das quatro dimensões em duas escalas: capacidade absortiva potencial e realizada, conforme sugerem Zahra e George (2002) e demais autores discutidos anteriormente. Entende-se que essa divisão é fundamental para avaliar a 
capacidade absortiva, pois pode permitir avaliar se a empresa possui uma capacidade absortiva maior para adquirir e assimilar conhecimento externo (potencial) ou para transformar e explorar o conhecimento adquirido (realizada). Para a escolha dos itens de mensuração da CA, as autoras levaram em consideração àqueles mais específicos ao perfil de empresas que buscam interagir com universidades. Assim, o instrumento de mensuração proposto serve para avaliar se as empresas que possuem interação com universidade detêm um nível de capacidade absortiva potencial que lhes permita adquirir e assimilar os conhecimentos gerados na relação das empresas com universidades, e de capacidade absortiva realizada, o que lhe permitirá transformar e explorar esses conhecimentos, gerando inovações. Esse instrumento de mensuração de CA foi aplicado em uma pesquisa survey com empresas que interagiam com universidades, conforme explicado na seção a seguir.

\section{Procedimentos Metodológicos}

\subsection{Coleta de dados para a realização da pesquisa survey}

Os dados analisados neste artigo foram coletados em uma pesquisa survey junto a empresas industriais localizadas no RS, utilizando-se o instrumento mencionado acima que serve para mensurar a capacidade absortiva (potencial e realizada e suas dimensões) das empresas investigadas. O survey foi realizado em empresas informadas pelos líderes dos grupos de pesquisa no Censo de 2010 do Diretório de Grupos de Pesquisa (DGP) do CNPq que realizaram interações com estes. Optou-se por focar nas empresas que interagiam com grupos das áreas de Engenharia Mecânica, de Materiais e Metalúrgica das Universidades do RS, visto que a área das Engenharias nesse estado é que mais possui grupos que declararam ter interação com empresas, segundo o Censo de 2010. A população da pesquisa era de 71 empresas privadas localizadas no RS de pequeno, médio e grande porte, conforme classificação do SEBRAE. Os respondentes foram identificados previamente por meio de contato telefônico e eram profissionais envolvidos com atividades de inovação das empresas e que participavam (ou tinham participado) do processo de interação com a(s) universidade(s).

O instrumento elaborado para a coleta dos dados foi submetido à avaliação na etapa de préteste, na qual foram entrevistadas 13 empresas. O questionário estruturado foi organizado em três partes: elementos da capacidade absortiva (CA), características da interação universidade- empresa e informações a respeito da atividade de inovação da empresa. Para capturar as respostas referentes à CA foi utilizada uma escala do tipo Likert de concordância de 5 pontos, seguindo sugestões de estudos anteriores como Jiménez-Barrionuevo et al. (2011) e Camisón e Forés (2010). Após a validação do instrumento, foi aplicado o questionário em toda a população de empresas. A pesquisa ocorreu no período entre janeiro e março de 2013. O total de respostas foi de 32, representando $45 \%$ da população. As empresas da amostra foram classificadas conforme a CNAE 2.0, em que $84 \%$ destas pertencem à indústria de transformação, com a maior porcentagem de firmas inseridas no setor de fabricação de veículos automotores, reboques e carrocerias, representando $25 \%$ do total da amostra.

\subsection{Análise dos dados}

A Qualitative Comparative Analysis (QCA) é uma técnica de pesquisa qualitativa desenvolvida por Charles Ragin (1987), que permite fazer inferências causais, especialmente quando a base amostral não é grande. A técnica usa a lógica binária da Álgebra Booleana e permite interpretar os dados qualitativamente e, ao mesmo tempo, buscar relações de causalidade entre variáveis. Esse método pode ser classificado em dois níveis: um primeiro, epistemológico relacionado a como definir uma estratégia de pesquisa; e um segundo, relativo ao uso de técnicas concretas. Ou seja, a QCA é uma forma de confrontar dados e base teórica.

Em termos de técnicas, a QCA é um termo guarda-chuva que abriga quatro classificações 
de técnicas:

a) quando se referindo explicitamente à versão original (Booleana) de QCA, usa-se a notação csQCA (onde 'cs' significa 'crisp set' - conjunto numérico tradicional);

b) quando se referindo explicitamente à versão que permite condições de multicategoria, usa- se a notação mvQCA (onde 'mv' significa 'multi-value');

c) quando se referindo explicitamente à versão de conjuntos fuzzy, usa-se fsQCA (onde 'fs'significa 'fuzzy set'); e

d) quando se referindo a fuzzy set, significa designar a análise fuzzy set original, conforme desenvolvida por Ragin (2000).

Este artigo tem como base metodológica a abordagem de conjuntos fuzzy (fuzzy set). Um conjunto fuzzy é definido matematicamente por meio da atribuição de um valor que representa o grau de pertinência ao conjunto de cada indivíduo no universo estuado. Este grau de pertinência representa a semelhança deste indivíduo ao significado que dá identidade ao conjunto. Em termos formais tem-se: um conjunto fuzzy $A$ é caracterizado por uma função de pertinência ${ }_{A}(x)$, a qual associa a cada elemento de um domínio, espaço ou um universo de discurso $X$, um número real no intervalo unitário $[0,1]$, com o valor de representando ${ }_{A}(x) \mathrm{em} X$ o grau de pertinência de $x$ em $A$ (ZADEH, 1965).

Assim, diferentemente de trabalhar com conjuntos a partir de variáveis binárias (característico de crisp set - Booleano), conjuntos fuzzy permitem inserir valores de associação entre ' 0 ' e ' 1 ' e não somente ' 0 ' ou' 1 '. Não se trata de uma mera transformação de uma variável binária em uma variável contínua, mas é antes uma calibração de um conjunto de variáveis relatadas a um grau de pertencimento dentro de uma categoria. Através do processo de calibragem, uma variável (ou múltiplas variáveis) relacionam-se ao(s) conjunto(s) fuzzy, e permitem uma avaliação qualitativa e quantitativa de um determinado estudo. Em outras palavras, ao invés de se atribuir 0 ou 1 às variáveis, pode-se recorrer a uma escala entre 0 e 1 , indicando a variação entre "nenhuma" e "total associação", respectivamente, das variáveis ao conjunto específico. Por exemplo: uma situação X (e.g. empresas com capacidade de absorção alta) pode ter diferentes graus de pertencimento a uma condição A (e.g. ter relacionamento com grupos de pesquisa de universidades), como: nenhum pertencimento $(0)$, baixa $(0,25)$, média $(0,5)$, alta $(0,75)$ ou total associação(1).

As funções de pertinência associadas aos conjuntos fuzzy dependem não somente do conceito a ser representado, mas também do contexto no qual são usadas. As funções podem ter diferentes formas e algumas propriedades, como continuidade, podem ser exigidas. A forma de pertinência, geralmente utilizada, é a função triangular descrita como segue (ilustrado na Figura 1):

Cada subconjunto qualitativo foi encarado como um número fuzzy triangular. A função de pertinência triangular de uma característica de qualidade $x$ é descrita como segue:

$\mu_{A}(x)=\left\{\begin{array}{c}0, x \leq \rho_{\min } \\ \frac{x-\rho_{\min }}{c-\rho_{\min }}, x \in\left(\rho_{\min }, c\right) \\ \frac{\rho_{\max }-x}{\rho_{\max }-c}, x \in\left(c, \rho_{\max }\right) \\ 0, x \geq \rho_{\max }\end{array}\right.$

sendo os valores de dispersão mínimo $\rho_{\min }$ e máximo $\rho_{\max }$, respectivamente, e o parâmetro $c$ o centro ou valor modal da função. 


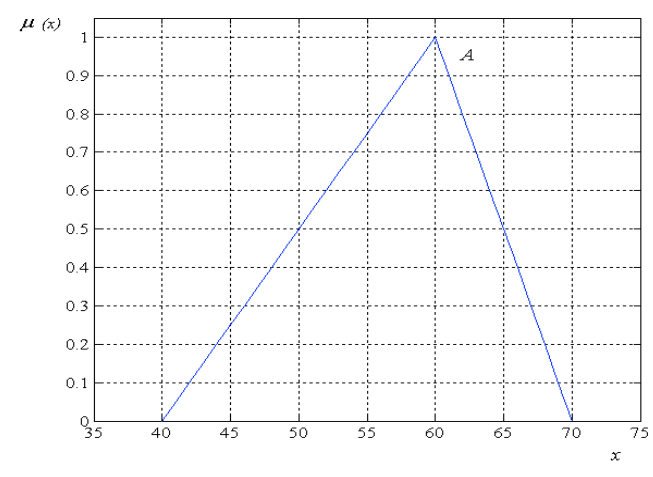

Figura 1: Função de Pertinência Triangular.

Fonte: Elaborado pelos autores.

Em termos configuracionais e metodológicos, a técnica fsQCA segue basicamente os seguintes princípios (RIHOUX; RAGIN, 2008):

1. Causalidade Complexa: assume-se que, frequentemente, é a combinação de condições e/ou circunstâncias que produz um fenômeno (um resultado). Isso quer dizer que a metodologia reconhece que todo fenômeno (especialmente o social) tem como causa uma combinação variada de condições, as quais não podem ser desassociadas ou isoladas.

2. Equifinalidade: ou seja, se assume que várias combinações diferentes de condições podem gerar o mesmo resultado. Por exemplo: em um caso, o evento $\mathrm{E}$ foi gerado pela combinação das condições $\mathrm{A}$ e $\mathrm{B}[\mathrm{AB} \rightarrow \mathrm{E}]$, mas, em outro caso, as causas $\mathrm{C}$ e $\mathrm{D}$ podem determinar o mesmo resultado $[\mathrm{CD} \rightarrow \mathrm{E}]$.

3. Sensível ao Contexto: Assume-se que dependendo da configuração contextual (ou do ambiente), uma determinada condição pode ter um impacto diferente sobre o resultado. Esse princípio é também chamado de princípio da multifinalidade, no qual determinadas condições

podem gerar ou contribuir para gerar resultados diferentes em contextos ou tempos distintos. Assim, $\mathrm{AB}$ podem gerar $\mathrm{E}[\mathrm{AB} \rightarrow \mathrm{E}]$, mas a ausência de $\mathrm{A}$ (a) combinado com $\mathrm{C}$ também pode gerar $\mathrm{E}[\mathrm{aC} \rightarrow \mathrm{E}]$. Desse modo, rejeita-se tanto qualquer forma de causalidade permanente, uma vez que a causalidade é relacionada ao contexto e à conjuntura.

4. Dados qualitativos: os dados estão associados aos graus de pertencimento aos conjuntos

As relações conceituais e inferenciais entre condição e resultado são baseadas na teoria dos conjuntos. Um estudo nessa perspectiva deve seguir cinco passos metodológicos: primeiro é a escolha dos conjuntos e seus dados qualitativos e quantitativos; segundo, deve-se - a partir de referências teóricas pertinentes -, estabelecer as relações de pertencimento entre os dados dos conjuntos escolhidos; terceiro, a partir de técnicas do fsQCA, são determinadas as configurações de causas/condições para a obtenção do resultado; quarto passo é o uso dos conceitos de consistência e cobertura para validar (ou não) as configurações encontradas; quinto passo é a interpretação do resultado.

Porém, é válido destacar que para se construir uma força explicativa robusta das relações causais regulares intra e/ou entre casos (condição $\rightarrow$ resultado), a fsQCA baseia-se em dois postulados epistemológicos fundamentais: a condição necessária e a condição suficiente. Uma 
condição é necessária para a ocorrência de um resultado [Y] se a condição está sempre presente quando [Y] ocorre. Ou seja, o resultado [Y] não ocorre na ausência desta condição. Uma condição é suficiente (mas não necessária) se ela é capaz de produzir um resultado [Y] por ela mesma, mas, ao mesmo tempo, o resultado [Y] também poderia ocorrer em função de outras condições presentes. Assim, o resultado [Y] pode advir não de uma única condição, mas de uma combinação de condições.

Além disso, as medidas de consistência e cobertura no âmbito do fsCQA avaliam a força (e/ou aderência) dos dados empíricos aos argumentos teóricos utilizados nas relações de conjunto estudados. Essa avaliação é importante porque o resultado encontrado pode advir não apenas de uma única relação causal específica; outras combinações de relações causais podem gerar o mesmo resultado. Assim, a medida de consistência mostra o subconjunto de casos relacionados à condição estabelecida e o resultado, no âmbito de todos os casos relacionados a essa condição. A medida de cobertura mostra a proporção de casos que contém a condição no total de número de casos nos quais o resultado está presente.

No $f s Q C A$, a medida de consistência é a proporção de casos onde os graus de pertencimento da condição são menores ou iguais aos graus de pertencimento do resultado no total dos casos relacionados à condição estabelecida. Altos valores na medida de consistência indicam que a condição é suficiente para o resultado. A medida de cobertura é a proporção de casos nos cujos graus de pertencimento da condição são menores ou iguais aos graus de pertencimento do resultado no total de número de casos, cujos graus de pertencimento do resultado são maiores que zero. Ou seja, esta medida mostra a importância de uma causa (ou uma combinação de causas) para a obtenção de um resultado. Se existem diversos "caminhos" para um mesmo resultado, a medida de cobertura para qualquer relação causal deve ser pequena.

\section{Descrição e análise dos dados}

Nesta seção é descrito como são obtidas e testadas as condições necessárias e suficientes para uma empresa apresentar capacidade absortiva alta (CAAlta), segundo uma transcrição das respostas dos entrevistados para conjuntos fuzzy.

Cada partição teórica de capacidade absortiva - capacidade de aquisição, capacidade de aquisição, capacidade de transformação e capacidade de exploração - foi investigada mediante questionário em escala Likert de cinco opções. Para cada partição houve 5 ou 6 perguntas. Obtevese uma média dos valores Likert de cada partição para os diferentes entrevistados. A seguir, cada partição teórica foi construída como conjuntos fuzzy. Por exemplo, a capacidade de aquisição foi classificada em três conjuntos - Capacidade de Aquisição Baixa (caaqbaixa), Capacidade de Aquisição Média (caaqmédia) e Capacidade de Aquisição Alta (caaqalta). Os pontos referenciais para definir os intervalos "alta", "média" e "baixa" na escala Likert baseiam-se no trabalho de Rosa (2103, p. 106, Quadro 12), que estabeleceu Capacidade Absortiva Alta nos valores acima de 3,6 para os dados coletados no surve ${ }^{7}$. Neste sentido, ao valor de 3,5 na escala Likert foi designado o grau de pertencimento 0 para o conjunto "CAAlta", cujo gradiente eleva-se até o grau de pertencimento 1 para o valor 5 da escala Likert. Obedecendo o princípio de sobreposição dos conjuntos inerente à teoria dos conjuntos fuzzy, o valor de 3,5 Likert também referenciou o conjunto "CA Média" - com grau de pertencimento 1, e de "CA Baixa", com grau de pertencimento 0 .

A variável de resultado Capacidade Absortiva (CA) também foi representada nas nuances CABaixa, CAMédia e CAAlta. Os conjuntos fuzzy foram propostos como funções triangulares

\footnotetext{
${ }^{7} \mathrm{O}$ questionário do survey foi avaliado como consistente pelo teste Alfa de Cronbach (ROSA, 2013).
} 
conforme a Figura 2.

Os valores médios de cada partição teórica de capacidade absortiva de cada respondente foram aplicados às funções que compõem os conjuntos da Figura 2. Desta forma obteve-se o grau de pertencimento da respectiva média ao correspondente conjunto baixa, média ou alta de Capacidade de Aquisição, Capacidade de Assimilação, Capacidade de Transformação e Capacidade de Exploração - todas condições antecedentes -, e de Capacidade Absortiva (CA) da empresa, variável consequente. Os graus de pertencimento obtidos por este processo estão dispostos na Tabela 1.

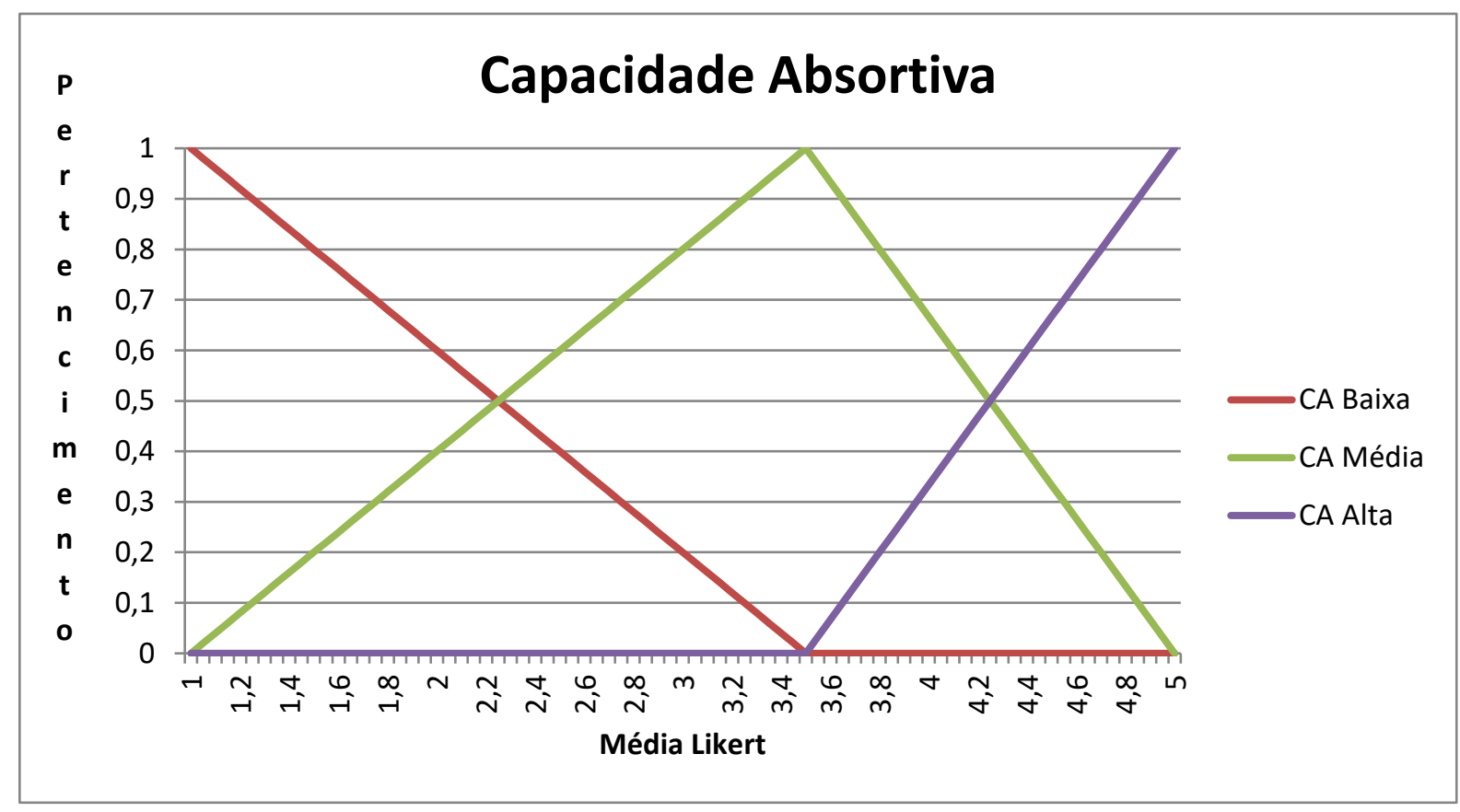

Figura 2 - Conjuntos Fuzzy de Capacidade Absortiva

Fonte: elaborado pelos autores

Uma vez formatados os pertencimentos das respostas das empresas aos conjuntos fuzzy, utilizou o software livre $f_{s} Q C A$ para descrever condições necessárias e suficientes para alcançar Capacidade de Absorção Alta.

Em um primeiro processamento, exploraram-se combinações dos conjuntos antecedentes de Capacidade de Aquisição (Alta e Média), Capacidade de Assimilação (Alta e Média), Capacidade de Transformação (Alta e Média) e Capacidade de Exploração (Alta e Média) para alcançar Capacidade Absortiva Alta. As variantes dos conjuntos "Baixa" poderão ser averiguadas em outra oportunidade mediante o uso da mesma base de dados.

Para estabelecer as combinações causais para CA Alta, acionou-se a opção Fuzzy Truth Table Algorithm do $f_{s} Q C A$. A resultante é uma lista de combinações para alcançar a variável CA Alta. Nesta lista, cada conjunto antecedente é representado como um vetor: quando é condição relevante, o valor da célula é 1; quando o conjunto não participa da combinação explicativa, o valor da célula é zero ${ }^{8}$. Além da lista de combinações possíveis, já é gerada a Consistência de cada combinação.

\footnotetext{
${ }^{8}$ Esta apresentação binária não é uma utilização clássica (crisp) dos conjuntos, é apenas uma indicação de qual vetor (ou conjunto) foi participante da configuração que resultou em CA Alta. Os graus de pertencimento processados pelo software são os constantes na Tabela 1.
} 
Tabela 1 - Grau de pertencimento das médias das respostas obtidas no survey

\begin{tabular}{|c|c|c|c|c|c|c|c|c|c|c|c|c|c|c|c|}
\hline Emnresa & CAaqBaixa & CAaqMédia & CAaqAlta & CAasBaixa & CAasMédia & CAasAlta & CAtrBaixa & CAtrMédia & CAtrAlta & CAexBaixa & CAexMédia & CAexAlta & CAbaixa & CAmédia & CAalta \\
\hline 1 & 0 & 0.76 & 0.24 & 0.03 & 0.97 & 0 & 0 & 0.86 & 0.14 & 0.03 & 0.97 & 0 & 0 & 0.93 & 0.07 \\
\hline 2 & 0,2 & 0,8 & 0 & 0 & 0,48 & 0,52 & 0 & 0,76 & 0,24 & 0 & 0,95 & 0,05 & 0 & 0,88 & 0,12 \\
\hline 3 & 0 & 0.76 & 0.24 & 0.09 & 0.91 & 0 & 0.09 & 0.91 & 0 & 0 & 0.76 & 0.24 & 0 & 0.95 & 0.05 \\
\hline 4 & 0 & 0.57 & 0.43 & 0 & 0.1 & 0.9 & 0 & 0.57 & 0.43 & 0 & 0.67 & 0.33 & 0 & 0.48 & 0.52 \\
\hline 5 & 0 & 0.67 & 0.33 & 0 & 0.95 & 0.05 & 0.03 & 0.97 & 0 & 0 & 1 & 0 & 0 & 0.92 & 0.08 \\
\hline 6 & 0 & 0.57 & 0.43 & 0 & 0.76 & 0.24 & 0.2 & 0.8 & 0 & 0 & 0.76 & 0.24 & 0 & 0.86 & 0.14 \\
\hline 7 & 0 & 0.57 & 0.43 & 0 & 0.57 & 0.43 & 0 & 0.86 & 0.14 & 0 & 0.48 & 0.52 & 0 & 0.62 & 0.38 \\
\hline 8 & 0 & 0.76 & 0.24 & 0 & 0.76 & 0.24 & 0.03 & 0.97 & 0 & 0 & 0.86 & 0.14 & 0 & 0.86 & 0.14 \\
\hline 9 & 0 & 0.86 & 0.14 & 0 & 0.76 & 0.24 & 0 & 0.57 & 0.43 & 0.37 & 0.63 & 0 & 0 & 0.95 & 0.05 \\
\hline 10 & 0 & 0.57 & 0.43 & 0 & 0.86 & 0.14 & 0 & 0.67 & 0.33 & 0 & 0.67 & 0.33 & 0 & 0.69 & 0.31 \\
\hline 11 & 0 & 0.29 & 0.71 & 0 & 0.19 & 0.81 & 0 & 0.95 & 0.05 & 0 & 0.57 & 0.43 & 0 & 0.5 & 0.5 \\
\hline 12 & 0.14 & 0.86 & 0 & 0 & 0.86 & 0.14 & 0.03 & 0.97 & 0 & 0 & 0.56 & 0.44 & 0 & 0.92 & 0.08 \\
\hline 13 & 0 & 0.38 & 0.62 & 0 & 0.48 & 0.52 & 0 & 0.29 & 0.71 & 0 & 0.57 & 0.43 & 0 & 0.43 & 0.57 \\
\hline 14 & 0 & 0.56 & 0.44 & 0 & 0.57 & 0.43 & 0 & 0.4 & 0.6 & 0 & 0.95 & 0.05 & 0 & 0.62 & 0.38 \\
\hline 15 & 0.2 & 0.8 & 0 & 0 & 0.76 & 0.24 & 0.03 & 0.97 & 0 & 0 & 0.67 & 0.33 & 0 & 0.95 & 0.05 \\
\hline 16 & 0 & 0.38 & 0.62 & 0 & 0.57 & 0.43 & 0 & 0.19 & 0.81 & 0 & 0.67 & 0.33 & 0 & 0.45 & 0.55 \\
\hline 17 & 0 & 0.67 & 0.33 & 0 & 0.67 & 0.33 & 0 & 0.48 & 0.52 & 0 & 0.67 & 0.33 & 0 & 0.62 & 0.38 \\
\hline 18 & 0 & 0.67 & 0.33 & 0 & 0.95 & 0.05 & 0 & 0.76 & 0.24 & 0 & 0.53 & 0.47 & 0 & 0.73 & 0.27 \\
\hline 19 & 0 & 0.95 & 0.05 & 0 & 0.86 & 0.14 & 0 & 0.76 & 0.24 & 0 & 0.38 & 0.62 & 0 & 0.74 & 0.26 \\
\hline 20 & 0 & 0.95 & 0.05 & 0 & 0.11 & 0.89 & 0 & 0.67 & 0.33 & 0 & 0.48 & 0.52 & 0 & 0.55 & 0.45 \\
\hline 21 & 0.14 & 0.86 & 0 & 0 & 0.19 & 0.81 & 0 & 0.57 & 0.43 & 0 & 0.19 & 0.81 & 0 & 0.55 & 0.45 \\
\hline 22 & 0 & 0.95 & 0.05 & 0 & 0.38 & 0.62 & 0 & 0.57 & 0.43 & 0 & 0 & 1 & 0 & 0.48 & 0.52 \\
\hline 23 & 0 & 0.48 & 0.52 & 0 & 0.67 & 0.33 & 0 & 0.76 & 0.24 & 0 & 0.57 & 0.43 & 0 & 0.62 & 0.38 \\
\hline 24 & 0 & 0.57 & 0.43 & 0 & 0.67 & 0.33 & 0 & 0.95 & 0.05 & 0 & 0.38 & 0.62 & 0 & 0.64 & 0.36 \\
\hline 25 & 0 & 0.19 & 0.81 & 0 & 0.19 & 0.81 & 0 & 0.1 & 0.9 & 0 & 0.48 & 0.52 & 0 & 0.24 & 0.76 \\
\hline
\end{tabular}


Uma vez estabelecidas as combinações necessárias e suficientes para obter CA Alta, bem como a respectiva consistência, restou estabelecer a Cobertura de cada possibilidade. Utilizou-se a seguinte fórmula, conforme proposto em Ragin (2006, p. 300):

$$
\text { Cobertura }(X i \leq Y i)=\sum(\min X i, Y i) / \sum(Y i)
$$

na qual $X i$ represente o pertencimento aos conjuntos antecedentes e $Y i$ a o pertencimento a CA Alta, para firmas $i=1$ a 25 .

A Tabela 2 apresenta os resultados da descrição de condições necessárias e suficientes para alcançar Alta Capacidade Absortiva decorrentes do processamento das respostas de vinte e cinco empresas.

Considerando valores mínimos para avaliação de 0,8 para Consistência e de 0,5 para Cobertura, há duas combinações importantes para uma empresa desenvolver uma Capacidade Absortiva elevada. Na combinação da linha 5 a CA Alta implica uma Capacidade de Aquisição Média $e$ Capacidade de Assimilação Alta $e$ Capacidade de Transformação Média $e$ Capacidade de Exploração Média. Já a combinação da linha 8 revela outro caminho para alcançar um CA Alta e que implica Capacidade de Aquisição Alta e Capacidade de Assimilação Alta $e$ Capacidade de Transformação Média $e$ Capacidade de Exploração Média.

Tabela 2 - Condições Necessárias e Suficientes para CA Alta

\begin{tabular}{c|c|c|c|c|c|c|c|c|c|c|c}
\hline & aqméd & aqalt & asméd & asalt & trméd & tralt & exméd & exalt & caalta & Consis & Cober \\
\hline 1 & 1 & 0 & 1 & 0 & 1 & 0 & 1 & 0 & 1 & 0,44 & 0,60 \\
\hline 2 & 1 & 0 & 1 & 0 & 1 & 0 & 0 & 1 & 1 & 0,70 & 0,56 \\
\hline 3 & 1 & 0 & 0 & 1 & 1 & 0 & 0 & 1 & 1 & 0,06 & 0,62 \\
\hline 4 & 1 & 0 & 1 & 0 & 0 & 1 & 1 & 0 & 1 & 0,80 & 0,46 \\
\hline $\mathbf{5}$ & $\mathbf{1}$ & $\mathbf{0}$ & $\mathbf{0}$ & $\mathbf{1}$ & $\mathbf{1}$ & $\mathbf{0}$ & $\mathbf{1}$ & $\mathbf{0}$ & $\mathbf{1}$ & $\mathbf{0 , 8 0}$ & $\mathbf{0 , 6 3}$ \\
\hline 6 & 0 & 1 & 1 & 0 & 1 & 0 & 1 & 0 & 1 & 0,72 & 0,50 \\
\hline 7 & 0 & 1 & 1 & 0 & 0 & 1 & 1 & 0 & 1 & 0,93 & 0,42 \\
\hline $\mathbf{8}$ & $\mathbf{0}$ & $\mathbf{1}$ & $\mathbf{0}$ & $\mathbf{1}$ & $\mathbf{1}$ & $\mathbf{0}$ & $\mathbf{1}$ & $\mathbf{0}$ & $\mathbf{1}$ & $\mathbf{0 , 9 0}$ & $\mathbf{0 , 5 0}$ \\
\hline 9 & 0 & 1 & 0 & 1 & 0 & 1 & 1 & 0 & 1 & 0,96 & 0,43 \\
\hline 10 & 0 & 1 & 0 & 1 & 0 & 1 & 0 & 1 & 1 & 1 & 0,36 \\
\hline
\end{tabular}

Fonte:Elaborado pelos autores 
Uma vez estabelecidas combinações causais necessárias e suficientes para obter uma capacidade absortiva alta, pode-se explorar quais as variações qualitativas das variáveis antecedentes têm maior possibilidade de atuarem como causas necessárias. Proceder desta forma, de trás para a frente (procurar variáveis necessárias depois de estabelecer as combinações necessárias e suficientes) após o uso do Fuzzy Truth Table Algorithm do fsQCA reduz o trabalho de processamento 9 . Investigou-se apenas a necessidade dos conjuntos implicados nas combinações causais das linhas 5 e 8 da Tabela 2, ou seja, aquisição média, aquisição alta, assimilação alta, transformação média e exploração média. Para tanto, utilizou-se a opção Necessary Conditions do fsQCA. Os níveis mínimos de consistência e de cobertura exigidos para selecionar as condições necessárias permaneceram 0,8 e 0,5 , respectivamente. Resultaram necessários os conjuntos assimilação alta, com consistência 0,88 e cobertura 0,71 , e a combinação de dois conjuntos aquisição alta e assimilação alta que, juntos, obtiveram consistência 0,98 e cobertura 0,65 . As duas condições são necessárias e insuficientes, ou seja, apenas combinadas com outros conjuntos das linhas 5 e 8 (tabela 1) geram CA Alta.

\section{Considerações Finais}

Os dados primários coletados com empresas que interagem com universidades em processo inovativos e analisados comparativamente através de pertencimento a conjuntos qualitativos (fuzzy sets), apresentou dois caminhos explicativos consistentes e importantes para obter capacidade de absorção alta. As empresas com elevada capacidade de absorção não são boas em todos os componentes, combinam pertencimentos a conjuntos "médios" com outros a "altos". Os pontos comuns na comparação entre estas duas combinações necessárias e suficientes para Capacidade de Absorção Alta são: a) que ambas combinam conjuntos antecedentes qualificados como "altos" (assimilação alta; ou aquisição alta e assimilação alta) com outros com pertencimento a "médios" (aquisição, transformação e exploração média; ou transformação e exploração média); $b$ ) assimilação alta aparece como um requisito importante nas duas combinações e é o conjunto com maior consistência e cobertura para ser necessário a uma alta capacidade absortiva.

As duas combinações consistentes para alcançar CA Alta sinalizam ser fundamental para boa absorção de conhecimento das empresas consultadas a elevada Capacidade de Aquisição, ou seja, a habilidade de localizar, identificar, avaliar e adquirir conhecimento externo importante para o desenvolvimento de suas operações; e a elevada Capacidade de Assimilação que consiste em conseguir compreender o conhecimento trazido de fora da organização. Por outro lado, uma vez alcançada elevada competência de assimilação ou de aquisição e assimilação, as habilidades de transformação - combinar e transferir o conhecimento assimilado-, e exploração - a incorporação do conhecimento em rotinas -, das mesmas empresas podem ser medianas. O estudo sinaliza, para o grupo de empresas avaliado, que a Capacidade Potencial elevada e combinada com Capacidade Realizada média implica CA Alta.

O tipo de mapeamento decorrente deste estudo é de interesse para estratégias empresariais. Dado que as respostas são de pessoal qualificado nas empresas, a informação mescla aporte teórico com experiências atreladas a trajetórias vividas no ambiente concorrencial.

O processamento efetuado neste estudo pode vir a ser complementado pela incorporação

\footnotetext{
${ }^{9}$ Investigar primeiramente as Condições Necessárias implicaria explorar 162 combinações $(\mathrm{C} 8,1$; C8,2; C8,3 e $\mathrm{C} 8,4)$. Ao concentrar a atenção apenas nas combinações necessárias e suficientes, o número de combinações testadas para necessidade reduziu para 30.
} 
dos conjuntos de qualidade "baixa", e os complementos de cada conjunto qualitativos ("nãobaixa", "não-média" ou "não-alta"). Isto abre espaço, por exemplo, para testar o conjunto fuzzy consequente Capacidade Absortiva Não-Alta e assim perceber combinações que afastam as empresas de um caminho inovativo bem sucedido.

As informações coletadas para outro processamento foram reaproveitadas para a presente discussão. A tradução da "média Likert" em pertencimento a fuzzy sets merece maior reflexão. Isto está além do escopo deste esforço inicial. $\mathrm{O}$ uso de questionários especificamente talhados para o método comparativo e qualitativo é uma opção a considerar.

Por fim, entende-se que a relevância desse método é que ele permite a compreensão da capacidade de absorção (CA) de empresas que possuem interação com universidades de uma forma mais flexível e ponderada pelos fatores mais relevantes para a melhor capacitação e absorção de conhecimentos pelas empresas. Nesse sentido pode também subsidiar políticas públicas que visem melhorar a CA e a competitividade das empresas nacionais, especialmente as que apresentam relacionamento com universidades.

\section{Referências bibliográficas}

ABRAMOVITZ, M. (1986), Catching up, forging ahead and falling behind, Journal of Economic History, No. 46, pp. 386-406

ABRAMOVSKY, L., et al. Understanding cooperative innovative activity: evidence from four European countries. Economics of Innovation and New Technology forthcoming, 2009.

CAMISÓN, C.; FÓRES, B. Knowledge absorptive capacity: new insights for its conceptualization and measurement. Journal of Business Research , v. 63, n. 7, p. 707-715, Jul. 2010.

COHEN, W. M; LEVINTHAL, D. A. Innovation and Learning: the two faces of R\&D. The Economic Journal, 99, p. 569-596, 1989.

. Absorptive-Capacity - a New Perspective on Learning and Innovation. Administrative Science Quarterly, v. 35, n. 1, p. 128-152, 1990.

Fortune Favors the Prepared Firm. Management Science, v. 40, n. 2, p. 227-251, 1994.

COSTA, A. B. et al. Interação universidade-empresa no Rio Grande do Sul: o caso do Programa de Pós-Gradação em Engenharia de Minas, Metalúrgica e Materiais da Universidade Federal do Rio Grande do Sul in SUZIGAN, W.; ALBUQUERQUE, E. M; CARIO, S.F., (Orgs). Em busca da inovação: interação universidade-empresa no Brasil. Belo Horizonte: Autêntica Editora, 2011: 199-334.

CULLAR, M. J; GALLIVAN, M. J. A framework for ex ante project risk assessment based on absorptive capacity. Elsevier, 173: 1123-1138, 2006.

DYER, JH and SINGH, H. The relational view: cooperative strategy and sources of interorganizational competitive advantage. Acad Manag Rev; 23(4):660-79, 1998.

GUSMÃO, M. R. P. Práticas e políticas internacionais de colaboração ciência-indústria. Revista Brasileira de Inovação, Rio de Janeiro, v. 1, n. 2, p. 327-360, jul./dez. 2002. Disponível em: http://ocs.ige.unicamp.br/ojs/index.php/rbi/article/viewArticle/247. Acesso em: 04 out. 2016.

FLATTEN, T. C, et al. A measure of absorptive capacity: scale development and validation. ESCP Europe, p. 98-116, 2011. 
GEORGE, G., S. ZAHRA, WOOD Jr. R. The effects of business-university alliances on innovative performance: a study of publicly traded biotechnology companies. Journal of Business Venturing 17(577-609), 2002.

JANSEN, Justin J.P; VAN DEN BOSCH, Frans A.J.; VOLBERDA, Henk W. Managing potential and realized absorptive capacity: how do organizational antecedents matter? Academy of Management Journal, v.48, n.6, p.999-1015, 2005.

JIMÉNEZ-BARRIONUEVO, et al. Validation of an instrument to measure absorptive capacity. Technovation, p. 190-202, 2011.

KIM L. Crisis construction and organizational learning: capability building in catching-up at Hyundai Motor. Organ Sci; 9(4):506-21, 1998.

LANE, P.J.; LUBATKIN, M. Relative absorptive capacity and inter-organizational learning. Strategic Management Journal, 19, 461-477, 1998.

LANE, P.J; SALK, J. E; LYLES, M., A. Absorptive capacity, learning, and performance in international joint ventures. Strategic Management Journal. V.22, p. 1139-1161, 2001.

LANE, P. J., et al. The Reification of Absorptive Capacity: A Critical Review and Rejuvenation of the Construct. Academy of Management Review 31(4): 833-63, 2006.

LAURSEN, K.; A.J. SALTER. Searching high and low: what types of firms use universities as a source of innovation?" Research Policy, 33: 1201-1215, 2004.

LEWANDOWSKA, M.S. (2015), Capturing Absorptive Capacity: Concepts, Determinants, Measurement Modes and Role in Open Innovation. International Journal of Management and Economics. No. 45, January-March 2015, pp. 32-56.

LINK, A. L; J. REES. Firm size, university based research, and the returns to R\&D. Small Business Economics, 2: 25-31, 1990.

MANSFIELD, E., 1991. Academic research and industrial innovation. Research Policy 20, $1-22$.

MOWERY, D.C.; OXLEY J.E. Inward technology transfer and competitiveness: the role of national innovation systems. Cambridge Journal of Economy; 19:67-93, 1995.

NELSON, R.R. Institutions supporting technical advance in industry. The American Economic

Review, vol. 76, $\mathrm{N}^{\mathrm{o}}$ 2, Papers and Proceedings of the Ninety- Eighth Annual Meeting of the American Economic Association, 1986, pp. 186-189.

PÓVOA, L. A Crescente Importância das Universidades e Institutos Públicos de Pesquisa no Processo de Catching-up Tecnológico. Revista de Economia Contemporânea. Rio de Janeiro, v. 12, n.2, p.273-300, maio/agosto de 2008 .

RAGIN, Charles. The comparative method: Moving beyond qualitative and quantitative strategies. Berkeley, Los Angeles and London: Univ. of California Press. 1987.

Fuzzy-Set Social Science. University of Chicago Press, 2000.

Set Relations in Social Research: Evaluating Their Cosistency and Coverage.

Political Analysis 14 (3), pp. 290-310, 2006.

RAPINI, M. S.; RIGHI, H. M. Metodologia e Apresentação da Base de Dados do Censo 2004 do Diretório dos Grupos de Pesquisa do CNPq. In: SUZIGAN, W.; ALBUQUERQUE, E. M; CARIO, S. F., (Org.). Em busca da inovação: interação universidade-empresa no Brasil. Belo Horizonte: Autêntica, 2011.p. 45-74.

RIHOUX, B., RAGIN, C. (eds). Configurational Comparative Methods. Qualitative 
Comparative Analysis (QCA) and Related Techniques: Applied Social Research Methods. Sage. Thousand Oaks and London, 2008.

ROSA, A. C. Capacidade Absortiva de Empresas que Possuem Interação com Universidades. 2013. 162 f. Dissertação (Mestrado em Economia) - Programa de Pós-Graduação em Economia, Universidade do Vale do Rio dos Sinos (UNISINOS). São Leopoldo, 2013.

ROSA, A. C.; RUFFONI, J. Mensuração da Capacidade Absortiva de firmas que possuem interação com universidades. Revista Economia e Desenvolvimento, vol. 26, n. 1, 2014.

SPENDER, J.C. Industry recipe: The nature and sources of managerial judgement. Oxford: Basil Blackwell, pp. 240, 1989.

SZULANSKI, G. Exploring internal stickiness: impediments to the transfer of best practice within the firm. Strategic Management Journal, 17, 27-43, 1996.

TORODOVA, G, and DURISIN, B. Absorptive capacity: valuing a reconceptualization. Academy of Management Review, 32(3), 774-786, 2007.

WAALKENS, J. Innovation in Medium-Sized Architectural and Engineering Firms. Ph.D. diss. Groningen University, Groningen, The Netherlands, 2006.

VAN DEN BOSCH, F. A. J.; VOLBERDA, H. W.; DE BOER, M. Coevolution of Firm Absorptive Capacity and Knowledge Environment: Organizational Forms and Combinative Capabilities. Organization Science, v. 10, n. 5, p. 551-568, 1 out. 1999. Disponível em: <http://pubsonline.informs.org/doi/abs/10.1287/orsc.10.5.551>. Acesso em: 19 set. 2016.

VAN DEN BOSCH, F. A. J.; VAN WIJK, R.V.; VOLBERDA, H.W. Absorptive Capacity: Antecedents, models and outcomes. In: M. Easterby-Smith and M. Lyles (eds) The handbook of organizational learning and knowledge management. Oxford: Blackwell, 2003, p. 278-302.

VEGA-JURADO, J.; GUTIÉRREZ-GRACIA, A.; FERNÁNDEZ-DE-LUCIO, I. Analyzing the determinants of firm's absorptive capacity: beyond R\&D. R\&D Management, v. 18, n. 4,p. 392-405, 2008.

VERSIANI, A.F, et al. (2010). Mensuração da Capacidade Absortiva: até que ponto a literatura avançou? XXXIV Encontro da Associação Nacional de Programas de PósGraduação em Administração (ANPAD), Rio de Janeiro, 25-29, setembro de 2010.

ZADEH, Lotfi A. Fuzzy Sets. Information and Control (8), 1965.

ZAHRA, S. A.; GEORGE, G.. Absorptive Capacity: A Review, Reconceptualization, and Extension. Academy of Management Review, v. 24, n. 2, p. 185-203, 2002. 